\title{
Analysis on Mechanical Behavior of Typical Asphalt Pavement Structure based on the Tire Load Effect
}

\author{
Fuming Liu*, Liming Zhang, Hongyan Sun \\ Nanchang Institute of Technology, Nanchang, 330099, China \\ *Corresponding Author.
}

\begin{abstract}
:
Further development of modern science and technology has made more science and technology technologies used in the modern social and life practice, and much more is this with the road construction. National development cannot be separate from the convenient traffic facilities, and in the current stage, the asphalt pavement design specification in China requires the road construction by simulating the force of the vehicle on the pavement structure with the round uniformly distributed load under the supposition of continuous interlayer pavement structure. However, as time goes on, the disadvantages of such design specification have gradually exposed, in which different materials, construction schedule and weather conditions will result in the significant difference in the asphalt pavement construction, thus resulting in weak interlayer viscous structure of the pavement, and finally causing the road subsidence and cracking. For this reason, this article has analyzed the mechanical behavior of typical asphalt pavement structure on the basis of explaining the influencing factors of the tire on typical asphalt pavement pressure under the tire load effect, with the expectation that there will be more professional construction of the asphalt pavement.
\end{abstract}

Keywords: Tire load effect, Asphalt pavement, Structural mechanics, Behavior analysis.

\section{INTRODUCTION}

In recent years, with the acceleration of social and economic development, the road construction develops to the better direction. However, the road construction philosophy of "strong base and weak surface" has resulted in the asphalt pavement structure state in China, mostly with the semirigid base course asphalt pavement with high bearing capacity. Recently, due to the pavement destruction, such as pot holes and cracks, many scholars have made the study on this, hoping that the pavement design in China can on the better direction. Chen 
Shangjiang and Zhang Xiaoning analyzed the mechanical behavior of the inverted base course asphalt pavement structure based on finite element method, and thought with the increase of the axial load, the tensile stress of the inverted base course [1].

Asphalt pavement is nearly in the linear increment and is obviously smaller than the bottom tensile stress of the typical semirigid base course asphalt pavement structure, having better crack resistance [2]. Yan Kezhen and You Lingyun et al., raised the non-uniform distribution load function, material parameters and boundary conditions on the road pavement stress based on the comprehensive analysis on the design theory for most of the current pavement. The results have shown that, if the isotropic properties are obviously considered for the pavement design materials, this will underestimate the permanent damage of the pavement structure depression and cracking deformation, so in the pavement design, it is required to properly consider the horizontal isotropic characteristics of the materials [3]. Li Debin and Jiang Tingting made deep analysis on the mechanical behavior of deflection, tensile stress, compressive stress and shear stress of the pavement structure under the overload, made the elaborate analysis on the influence of the thickness in the bituminous structure pavement on the mechanical behavior of the pavement structure, and raised the reasonable structure layer thickness adaptable to the heavy-load trunk road [4]. Zhang Huaizhi and Ren Junda et al. thought that the viscoelastic properties of bituminous mixture have resulted in the nonsymmetric characteristics of the mechanical response curve. With the increase of the temperature and the decrease of the acceleration, there is the increase in the three-direction strains in the bottom of the asphalt layer, horizontal strain in the bottom of the semirigid base course and the response amplitude of the compressive stress in the top of the soil base [5]. NiuLiqiang though that the temperature fatigue effect is the main reason for the cracking of the asphalt pavement from up to down, and the tensile stress on the pavement surface has aggravated such cracking, after the pavement temperature field measurement, asphalt ageing test and the analysis on low-temperature bending strain and bending stiffness modulus of the bituminous mixture of different ageing extents [6].

As the road technology building design gradually becomes mature, the conditions for the construction of asphalt pavement also become constantly strict. Nowadays, most of the design structures of the typical asphalt pavement in China involve the study of the mechanical behavior of the vehicle on the pavement structure on the basis of simulating the round uniformly distributed load and supposing that the structure layers are completely continuous [7]. However, the complex distribution principle of the vehicle tire ground contact pressure, and the irregular ground contact area of the tire, have resulted in obvious non-uniformity of the acting force. In the construction of asphalt pavement, the construction material difference, and obvious difference in construction procedure and time quality control have made the binding layer of the asphalt pavement quite fragile, unable to achieve the continuity in the ideal status 
[8]. For this reason, in order to extend the use duration of asphalt pavement and strengthen the continuous status of the asphalt pavement, it has the great practical significance to study the mechanical behavior of the typical asphalt pavement structure based on the tire load effect.

\section{INFLUENCING FACTORS OF THE TIRE ON TYPICAL ASPHALT PAVEMENT PRESSURE UNDER THE TIRE LOAD EFFECT}

\subsection{Vehicle Tire Structural Characteristics}

Generally speaking, radial and diagonal tire can be used as the typical tire structure for the explanation of the tire characteristics. The diagonal tire bead has the extensible characteristics, so that the diagonal tire is very easy to enclose the pavement in a protruding manner, achieving good smoothness. But it is easily influenced by the load effect, causing the deformation and resulting in the increase of the friction force and rolling resistance, thus reducing the wear resistance capacity of the diagonal tire [9]. While the radial tire has poor extensibility of the tire bead, thus resulting in poor enclosure capacity. Its hard band also enables to the radial tire made of rubber to have strong wear resistance, thus it is hardly penetrated by sharp apparatuses.

\subsection{Vehicle Tire Tread Pattern}

Generally speaking, in order to ensure sufficient adhesion force, the tire is usually designed with sufficient patterns to guarantee the friction effect, and the number of the tire patterns is also significantly related to the wear resistance of the vehicle tire and vehicle vibration [10]. The design rule of vehicle tire tread pattern will be arranged according to the structure, type and use extent. Usually under such rule, there are five typical patterns, that is, nonsymmetric, longitudinal, horizontal, block and mixed patterns [11]. With these patterns, the pressure distribution during the contact of the vehicle tires with the asphalt pavement in the vehicle running is noncontinuous.

\subsection{Vehicle Tire Pressure}

There is very large influence with the ground contact pressure between asphalt pavement and vehicle tire pressure. The tire pressure can change the vertical stiffness of the tire during the ground contact of the vehicle, and the change of the vertical stiffness will further decide the size of the tire ground contact pressure, and result in the change of the ground contact distribution area of the tire [12]. When such load reaches certain level, the increase of the ground contact pressure of the vehicle with the asphalt pavement has the influence of positive 
correlation with the vehicle tire pressure. Besides, if the peak value of the asphalt pavement ground contact pressure changes, ground contact pressure will decrease with the increase of the vehicle tire pressure. So, it may be known that, the change of the vehicle tire pressure to the ground contact pressure will impose very significant influence on the mechanical behavior of the asphalt pavement [13].

\subsection{Vehicle Tire Load}

The ground contact pressure of the vehicle tire will change with the vehicle. When the tire pressure is fixed, the bigger the load, the closer the shape of the ground side looking like oval. On the contrary, such load change will make its shape similar to rectangle [14]. In addition, tire load and tire pressure are two major factors influencing the tire ground contact pressure, and vehicle tire loads and tire pressures under different types may have different influencing effects. Generally, the vehicle tire has large lateral extensibility and flexibility, thus resulting in different mechanical behaviors for the ground contact area of different tires [15]. The radial tire pressure has the positive correlation with the load, while the diagonal tire has the lateral stiffness and ground contact area smaller than that of radial tire, thus its tire load will have large influence on the ground contact pressure [16].

\section{ANALYSIS ON THE MECHANICAL BEHAVIOR OF TYPICAL ASPHALT PAVEMENT STRUCTURE BASED ON THE TIRE LOAD EFFECT}

\subsection{Pavement Structure Combination}

To study the mechanical behavior of typical asphalt pavement structure, this article carries out the experimental analysis by choosing typical asphalt pavement structure as the study object with Ever Stress FE software in combination with the engineering practice [17]. In addition, this article has chosen the medium-size freight truck as the reference model for vehicle load, and the mixed pattern as the vehicle tire pattern. Generally, the vehicle tire has the complex ground contact pressure distribution in the typical asphalt pavement structure, but the law inside can still be found out. Refer to Table I for the distribution characteristics of the tire load along the tire width and length directions.

\section{TABLE I. Tire load distribution}

\begin{tabular}{|l|l|l|l|l|}
\hline $\begin{array}{l}\text { Tire load } \\
\text { distribution type }\end{array}$ & $\begin{array}{l}\text { Tire load } \\
\text { distribution shape }\end{array}$ & $\begin{array}{l}\text { Single } \\
\text { load/KN }\end{array}$ & wheel & $\begin{array}{l}\text { Ground contact pressure } \\
\text { maximum /minimum }\end{array}$ \\
\hline
\end{tabular}


Article History: Received: 10 May 2021 Revised: 20 June 2021 Accepted: 18 July 2021 Publication: 31 August 2021

\begin{tabular}{|l|l|l|l|}
\hline \multirow{2}{*}{ Uniform distribution } & Rectangular & \multirow{2}{*}{25} & $0.6 / 0.6$ \\
\cline { 2 - 2 } & Round & \\
\hline \multirow{2}{*}{$\begin{array}{l}\text { Convex non-uniform } \\
\text { distribution }\end{array}$} & Convex parabola & & \\
\cline { 2 - 2 } & $\begin{array}{l}\text { Convex strip } \\
\text { Convex half-sine } \\
\text { wave }\end{array}$ & \multirow{2}{*}{20} & $1.3 / 0.7$ \\
\hline $\begin{array}{l}\text { Concave non-uniform } \\
\text { distribution }\end{array}$ & $\begin{array}{l}\text { Concave half-sine } \\
\text { wave }\end{array}$ & & \\
\cline { 2 - 2 } & Concave parabola & & \\
\hline
\end{tabular}

\subsection{Analysis Discussion}

Usually, the deflection value of the asphalt pavement is small under the vehicle tire load effect, and for further comparison of the details, this article has chosen the part zone under the tire load effect and chosen the corresponding maximum deflection $\mathrm{Uz}$ and the space spots. All are indicated in Table II.

TABLE II. Maximum deflection and maximum shear strain of pavement structureunder different tire load distribution

\begin{tabular}{|l|l|l|l|}
\hline $\begin{array}{l}\text { Tireload } \\
\text { distribution type }\end{array}$ & $\begin{array}{l}\text { Tireload } \\
\text { distribution shape }\end{array}$ & $\begin{array}{l}\text { Maximum shear } \\
\text { strain }\end{array}$ & Maximum deflection Uz \\
\hline \multirow{2}{*}{ Uniform distribution } & Rectangular & 455.8 & 38.9 \\
\cline { 2 - 4 } & Round & 533.7 & 39.5 \\
\hline \multirow{2}{*}{$\begin{array}{l}\text { Convex non-uniform } \\
\text { distribution }\end{array}$} & Convex parabola & 509.6 & 42.6 \\
\cline { 2 - 4 } & $\begin{array}{l}\text { Convex strip } \\
\text { Convex half-sine } \\
\text { wave }\end{array}$ & 488.3 & 41.8 \\
\hline \multirow{2}{*}{$\begin{array}{l}\text { Concave non-uniform } \\
\text { distribution }\end{array}$} & $\begin{array}{l}\text { Concave half-sine } \\
\text { wave }\end{array}$ & 736.2 & 42.1 \\
\cline { 2 - 4 } & Concave parabola & 735.9 & 38.8 \\
\hline
\end{tabular}

Different vehicle tire load distribution shapes have resulted in the difference of maximum shear strain and maximum deflection of the asphalt pavement, and such difference is reflected in the particularly significant in the tire load contact position, the longer distance from the contact position, the smaller chance for difference. For the same tire load distribution type, the difference caused by different tire load distribution shapes is not obvious. And for the 
maximum deflection value of the typical asphalt pavement, convex non-uniform distributions biggest, concave non-uniform distribution is smallest, and the uniform distribution is in the medium value. With rectangular uniform distribution as the benchmark, the convex half-sine wave non-uniform distribution value is significantly improved, while the concave stripped non-uniform distribution value has not been significantly changed. So, it can be basically confirmed that concave non-uniform distribution and uniform distribution have weak influence on the maximum value of road surface deflection of the typical asphalt pavement, and it can be further considered that for the same type of tire load distribution, there will also be different influence on the deflection of the typical asphalt pavement for different vehicle tires, but the influence on the maximum value will not be significant. In addition, from the position of the maximum deflection value on the road surface of the typical asphalt pavement, the tire load distribution shape will also result in the deflection of the happening point of the maximum deflection value of the typical asphalt pavement and be closer to the center point of the tire ground contact. If in the concave non-uniform distribution and uniform distribution, the maximum deflection value will move towards the center point of the tire ground contact center, where the concave non-uniform distribution is somewhat closer. While for the same type of tire load distribution, the change of the shape will not obviously change with the maximum deflection value of the pavement.

\section{BRIEF CONCLUSION}

In summary, road construction involves the heavy burden for national economic development, so strengthening the analysis on the mechanical behavior of asphalt pavement structure can provide better experience for the future road construction, and improve the quality of the asphalt pavement construction, and strengthen the use period and service life of the road construction. Additionally, the current flexible base course asphalt pavement has gradually become the important portion in the road construction, and it is possible to start from the vertical tire load of non-uniform distribution, for further st8udy on the mechanical change mechanism.

\section{ACKNOWLEDGMENT}

(1) The Science and Technology Fund Project of Jiangxi Provincial Transportation Department "Study on the structure, material design and construction of the asphalt pavement of the national provincial trunk highway under the climate of South China" (2017H0059)

(2) The science and technology fund project of Jiangxi Provincial Education Department "Study on asphalt pavement structure design and material composition optimization of concrete bridge surface in wet and hot and rainy areas" (GJJ151138). 


\section{REFERENCES}

[1] Chen S J, Zhang X N (2014) Mechanical behavior analysis of Inverted Asphalt Pavement Structure. Journal of architectural materials 17(4): 644-648

[2] Yan K Z, You L (2016) Mechanical behavior analysis of transversely isotropic asphalt pavement structure. Highway traffic science and technology 33(4): 1-6

[3] Li D B, Jiang T T (2014) Research on structural adaptability of heavy duty asphalt pavement based on structural combination characteristics. Road Machinery \& Construction Mechanization 31(7): 47-50

[4] Zhang H Z, Ren J D (2016) Measurement and Simulation of full-scale mechanical response of asphalt pavement structure. Journal of Harbin Institute of Technology 48(9): 41-48

[5] Li Q N (2017) Analysis of the causes of Top-Down cracks in asphalt pavement. Road Machinery \& Construction Mechanization 34(1): 69-72

[6] Shen A Q, Zhang Y H (2009) Comparative analysis of mechanical response of three kinds of asphalt pavement structures. Journal of CHANG'AN University: Natural Science Edition 29(4): 1-7

[7] Song Y, Ma S B (2015) Mechanical analysis of durable asphalt pavement structure at flexible base. Chinese and foreign highway 35(5): 94-97

[8] Tian Y G, Yan L L (2013) Influence of material modulus on mechanical performance of asphalt pavement pit repair structure. Journal of Wuhan University of Technology 35(11): 58-62

[9] Zhao Y Q, Yu X (2010) Influence of driving speed on mechanical parameters and response of asphalt pavement. Journal of Beijing University of Technology 36(9): 1253-1257

[10] Zhang J P, Wu S H (2013) Analysis of base plane contact state and pavement mechanical response based on shear elastic compliance. Highway traffic science and technology 30(1): 6-16

[11] MATT M Guide for mechanistic-empirical design of newand rehabilitation pavement structure IIIinois. USA: National Cooperative

[12] M.W. Witczak, K. Kaloush, T. Pellinen, H.V. Quintus, (2002) Simple performance test for Superpave mix design. NCHRP Report 465, D.C, National Academy Press, Washington

[13] Hao F, Liu Q T, Mo L T et al. (2017) Characterization of three-stage rutting development of asphalt mixtures. Construction and Building Materials 154: 340-348

[14] Wang J G, Molenaar André A A, M.F.C. van de Ven et al. (2018) Influence of internal structure on the permanent deformation behavior of a dense asphalt mixture et al. Construction and Building Materials 171:850-857

[15] Kolisoja P, Kalliainen A, N (2015) New mechanistic design approach for subgrade rutting of Low Volume Roads. Vuorimies Geotechnical Engineering for Infrastructure and Development: 301-306

[16] Christiane R, Ingrid C, Manfred N P (2017) Age in gand performance of warm mix asphalt pavements. Journal of Traffic and Transportation Engineering (English Edition) 4(04):388-394

[17] Du Y F, Chen J Q, Han Z et al. (2018) A review on solutions for improving rutting resistance of asphalt pavement and test methods. Construction and Building Materials 168: 893-905 